\title{
PROCEEDINGS OF THE SOCIETY OF BRITISH NEUROLOGICAL SURGEONS : 57th MEETING
}

The 57th meeting of the Society of British Neurological Surgeons was held in Holland as a joint meeting with the Netherlands Society of Neurosurgeons. Meetings were held at St. Ursula Clinic, Wassenaar, on May 9, and at the University Neurosurgical Department, Utrecht, on May 10. The two Presidents, W. R. Henderson (Leeds) and H. Verbiest (Utrecht), occupied the chair in rotation.

\section{Observations on Compression and Functional Recovery of Nervous Tissue \\ In 1936 A. C. DE VET (Wassenaar) reported a number} of clinical observations which seemed to suggest the existence of a special form of pressure transmission in the intracranial contents. This mechanism differed from that associated with a general rise of intracranial pressure and seen in most space-occupying lesions.

Homolateral symptoms and signs occurred in $12 \%$ of intracranial tumours (Kernohan and Woltman, 1928); it was generally accepted that they were caused by displacement of the pes pedunculus against the contralateral sharp edge of the tentorium (Magnan, 1878-79; Collier, 1904; Purves-Stewart, 1927; Groeneveld and Schaltenbrand, 1927; van der Drift, 1957). Such signs were based on a different mechanism and should not be considered as caused by tentorial herniation.

Homolateral affections of the basal nerves, especially the fifth, were most often seen in lesions situated in the upper parts of the cerebral hemispheres and were due to downward displacement of the hemisphere on to the skull base. This syndrome was seen most often with extracerebral lesions, such as meningiomata, haematomata, and so forth, and less often with intrinsic brain lesions. The reason for this was that intrinsic lesions, surrounded by soft brain tissue on all sides, exerted pressure in all directions and consequently the pressure in one direction was less than in the case of a meningioma which was fixed against the skull on one side and exerted considerable pressure towards the brain.

A mechanism similar to that of crus pressure was seen in the case of sphenoidal wing meningiomas which displaced the optic nerve against the opposite edge of the optic foramen and so caused a temporal hemianopia.

The mechanism of producing symptoms in the Morgagni-Morel syndrome was considered in some detail. It was considered that the hypertrophic bone in the frontal region not only damaged the underlying frontal lobes of the brain but also, by exerting downward pressure, damage was inflicted on the diencephalic portions of the brain by counter pressure against basal structures, bone or dura. It was suggested that this mechanism accounted for the symptoms of mental and endocrine disturbance and of involvement of basal nerves.

The case history of a patient was presented who had a hyperostosing meningioma of the left fronto-parietal region. He also suffered from left-sided trigeminal neuralgia which seemed to be of classical type. Removal of the tumour was followed by recovery of the trigeminal neuralgia. It was suggested that the neuralgia had been caused by counter pressure of the skull base against the trigeminal nerve.

It was stressed that symptoms caused by such chronic contre-coup mechanisms often suggest a localization of disease quite different from that of primary tumour and this may lead to faulty treatment. The value of electroencephalography in localization was stressed.

\section{REFERENCES}

Collier, J. (1904). St. Mary's Hosp. Gaz. (Lond.), 10, 54.

Drift, J. H. A. van der (1957). The Significance of Electro-encephalo graphy for the Diagnosis Localisation of Cerebral Tumours Leiden.

Groeneveld, A., and Schaltenbrand, G. (1927)." Dtsch. Z.Nervenheilk 97, 32 .

Kernohan, J., and Woltman, H. (1928). Proc. Mayo Clin., 3, 69.

Magnan (1878-79). Brain, 1, 562.

Purves-Stewart, Sir J. (1927). Intracranial Tumours and Some Errors in Their Diagnosis, p. 206. Oxford University Press, London

Vet, A. C. de (1936). Diagnostiek van het meningioma cerebr Amsterdam.

The Treatment of Prolonged Unconsciousness after Head Injury

A. v. d. ZWAN (Groningen) described a method of treating patients with prolonged unconsciousness and decerebrate rigidity after head injury. Only those cases with decerebrate rigidity were selected and comprised 11 patients. Unconsciousness in these cases was not very deep and seemed like normal sleep in contrast to lesions of the medulla which appeared to produce much deeper coma. The outstanding feature of the cases was decerebrate rigidity and mid-brain convulsions so that it was considered reasonable to treat them in the same way as status epilepticus.

Sedation was achieved with luminal or somnifane and when this was insufficient pentothal anaesthesia was used (eight cases). It was noted that experimental decerebrate rigidity in animals, produced by ligation of the carotids and basilar artery near the posterior cerebral, only appeared when anaesthesia had worn off.

Bronchial hypersecretion, so common in these cases, appeared to be reduced by this treatment and tracheotomy was required in only two cases. One of these had multiple rib fractures and a collapsed lung and the other was a late case admitted three weeks after injury from another hospital. 
If patients did not improve, or deteriorated, then exploratory burr holes were made. In five cases this operation disclosed a subdural collection of fluid over the whole surface of the brain. This fluid usually had a high protein content but was, nevertheless, considered by Professor Booy (The Free University, Amsterdam) to be cerebrospinal fluid. It was not thought to be equivalent to a subdural haematoma but to be a fluid collection all over the brain similar to those described by McConnell and Phillips at previous meetings of the Society. Such a transudation from the brain surface after head injury had been described by Luyendijk at a recent meeting of the Dutch Society. An interesting feature of the cases was that air pictures consistently failed to show any evidence of air in the posterior fossa.

All the patients survived. One remained unchanged and though he developed a sleep-wake mechanism no verbal contact was possible. Two cases were left demented and with gross neurological abnormalities but were attending a rehabilitation centre. One patient, although seriously demented and with a right spastic paresis, was able to earn his living. Of the remaining seven cases, one had only transient unconsciousness for 18 hours, and the other six were able to return to a normal life, one having tremor of the right arm and one being slightly demented.

In considering the pathogenesis of cerebral lesions after injury it was suggested that, although stretching and tearing of nerve fibres was probable (Strich, 1956), anoxia might also play a considerable part. Anoxia could occur during the period of shock and was probably present during mid-brain fits also in spite of the deep and rapid respirations. In this connexion it was noted that chlorpromazine therapy had been abandoned since it was known that this substance, even in low dosage, lowered the resistance of the mid-brain to stagnant anoxia.

It seemed possible also that brain movement during injury might result in stretching of vessels in the circle of Willis and subsequent vasoconstriction. The value of pentothal anaesthesia in producing vasodilatation during angiography was well known and perhaps this method of treatment played a valuable part in helping patients over a critical period.

It appeared that decerebrate rigidity was more often seen in children than in adults, and that their chances of recovery were greater. If stretching and tearing of nerve fibres was the cause of the lesion, then one might refer to the lesion in children as a "green-stick" fracture of the brain-stem.

\section{REFERENCE}

Strich, S. J. (1956). J. Neurol. Neurosurg. Psychiat., 19, 163.

\section{Extradural Haemorrhage}

Norman Whalley (Swansea) reviewed the findingsclinical and operative-and the end-results of 30 cases of extradural haemorrhage treated in the Neurosurgical Unit at Morriston Hospital, Swansea, during the period 1951-56. In this period 900 cases of injury to the head were admitted to the Neurosurgical, Traumatic, and Paediatric Units. The incidence of extradural haemorrhage was, therefore, $3.3 \%$. Five of the patients died, the recovery rate being approximately $82 \%$. Twenty-two patients were adults; eight were children of 12 years and under. Eighteen of the 22 adults recovered; seven of the children recovered.

In five cases there was no latent period. The average latent period in 25 cases was 11 hours. On admission to the Neurosurgical Unit, two-thirds of the cases showed alteration in consciousness together with pupillary changes. A similar proportion showed frank neurological abnormalities in the limbs. Five were in a state of decerebrate rigidity. Bradycardia was not a prominent feature in this series; it occurred in only eight cases. Only two patients presented with Jacksonian epilepsy.

Twenty-nine of these haemorrhages were localized accurately by the position of local scalp bruising. Fracture of the skull was present in 23 of the 30 cases; these fractures were seen either on radiographs or at operation. It was unusual to have other serious injuries in association with this condition. One patient had an injury to one optic nerve; another patient had a peripheral facial palsy; a third patient had a crush fracture of one dorsal vertebra without paraplegia.

Fifty per cent. of the haemorrhages were in the classical site in the temporal region.

The source of haemorrhage was found to be from the middle meningeal artery or its branches in 25 cases. In four cases bleeding came from the large venous sinuses (sagittal or lateral); in one case bleeding came from diploic channels at the fracture site.

The five cases which died were analysed in some detail. The first death occurred in a baby of 18 months. It was considered that death in this case was largely due to delay in starting a blood transfusion. The second death occurred in a young man of 22 years. He was transferred 24 hours after injury in a condition of decerebrate rigidity. A lumbar puncture had been performed immediately before his transfer and this was thought to have precipitated a tentorial pressure cone. The third death occurred in a man of 20 years. This case was transferred late and died in the ambulance. This patient was not operated upon. This patient also had a lumbar puncture before his transfer and this was thought to have precipitated tentorial herniation. The fourth death was in a man of 47 years who was transferred 48 hours after the injury. In this case there was also a small subdural haematoma. This patient had also been subjected to lumbar puncture but in this instance it did not result in tentorial herniation. It was felt that earlier operation would have resulted in recovery as there was no intrinsic brain damage apart from a small, superficial laceration of the temporal lobe. The fifth death occurred following a lumbar puncture. This was a man who had suffered from infrequent attacks of epilepsy for two years. More recently he developed headache and was admitted for investigation. There were no abnormal neurological signs and only slight blurring of the optic nerve heads. Following lumbar puncture a cerebellar pressure cone developed and the patient died within a matter of minutes. At necropsy an extradural clot was discovered in the posterior fossa. There was no fracture to be seen on radiographs and no fracture found at necropsy. Afterwards the relatives remembered that the patient had 
fallen out of bed during an epileptic attack 14 days before his admission, striking his head. No other lesion was found.

All the 25 survivors returned to work in jobs, in the home, or at school. Two patients died later; one four years later from pneumonia. This patient was the only one in the series who developed epilepsy. The second patient who died later developed a cerebral tumour two years after the operation for removal of the extradural haemorrhage.

The remaining 23 were all working. Two had permanent homonymous hemianopias. These were considered to be due to infarction of the occipital lobe secondary to kinking of the posterior cerebral artery over the sharp edge of the tentorium in the production of the tentorial herniation.

It was stressed that the diagnosis of extradural haemorrhage was essentially a clinical diagnosis. Lumbar puncture was an extremely dangerous procedure if there was the slightest possibility of surface clot compression of the brain. Radiographs of the skull were useful only if they could be obtained quickly; delay for any reason jeopardized the chances of survival. Local scalp bruising was one of the most valuable localizing signs.

Local anaesthesia was the method of choice when operating; any procedure which increased venous congestion of the brain might precipitate tentorial herniation. Unilateral dilatation and fixation of a pupil, while a valuable lateralizing sign, was invariably a late sign and one of danger, indicating that speedy measures would have to be taken to relieve pressure on the brain-stem. It was of the utmost importance to provide blood transfusion, particularly in young children.

\section{Atlanto-axial Dislocations}

C. H. LENSHOEK (Groningen) presented the clinical, radiological, and operative features in 11 cases of atlantoaxial dislocation. These were divided into three groups.

The first group comprised six cases of traumatic dislocation.with fracture of the odontoid. Two were in children of 5 years, the remainder adults. In the two children there was an epiphysial separation with forward dislocation of the atlas together with a Jefferson fracture of the atlas in two cases. Neurological complications were present in two cases, one of them severe. Treatment consisted of immobilization alone, skull traction followed by plaster, and in two cases spinal fusion.

The problem of traumatic subluxation was discussed briefly.

The second group consisted of two cases of nontraumatic acquired dislocations. One case was that of a 12-year-old boy with rheumatic arthritis; neurological signs were absent and spinal fusion was performed. The second case was that of a 75-year-old woman with cervical spondylosis and a quadriparesis who was treated by spinal cord decompression.

The third group consisted of three cases of occipitalization of the atlas with subluxation of the odontoid. Physiological and pathological movements of the odontoid were discussed briefly. Treatment consisted of decompression in two cases and decompression and fusion in the other.
Surgical treatment in this series was mostly carried out with the patient in the sitting position.

\section{Endaural Hernia Cerebri}

Peter H. SchurR (London) presented the case histories of four patients with endaural cerebral hernia. He noted that cerebral hernia was one of several conditions, once relatively familiar and now becoming rare. However, a cryptic form still occurred occasionally which was the more important because of the readiness with which it responded to treatment. In this form there was an endaural prolapse of brain tissue through the roof of the ear cavity after mastoidectomy which, though sometimes visible at the auditory meatus, might only be visible on auriscopy or after dividing a false membrane which sometimes formed across the area.

Case 1.-F.H., a man, 48 years old, had chronic suppurative otitis media. During an endaural mastoidectomy the dura of the middle fossa was torn. Recovery was uneventful but the ear continued to discharge. Six months after the operation a pulsatile swelling was seen in the aural cavity. During the next six months it grew large enough to be seen at the meatus, after which it diminished in size. During this time the discharge continued. Craniotomy revealed an opening in the durat through which the brain had herniated. This was covere with fascia lata, after which the endaural swelling disco appeared and the discharge ceased.

Case 2.-B. H., a woman, 29 years of age, had suffere from otitis media seven years previously for which $\$$ radical mastoidectomy had been performed by the post auricular route. An offensive discharge returned an $\Phi$ continued intermittently despite treatment. Furthes $\stackrel{9}{+}$ explorations were made following the development of $\vec{f}$ temporal lobe epilepsy, headache, and temporal pain Finally after dividing a web crossing the mastoid cavity, $\infty$ an endaural cerebral hernia was seen. This was repaired with temporal fascia through a craniotomy, following which the swelling disappeared, the fits almost ceased, and the remaining symptoms cleared up.

Case 3.-R.S., a man, aged 54 years, suffered a persistent aural discharge after a radical mastoidectomy and destruction of the labyrinth. A pulsatile swelling was seen in the roof of the cavity and repaired by $\mathrm{Mr}$. Julian Taylor, who used a similar technique. Two months after the operation the protuberance had gone.

Case 4.-M.A., a 15-year-old girl, with a seven-year history of otorrhoea, had an endaural modified radical mastoidectomy performed. Convalescence was uneventful until eight months after the operation when a swelling appeared in the ear cavity and increased in size until it eventually filled the meatus. This was followed by cerebrospinal fluid otorrhoea and headache. Mr. Julian Taylor repaired the hernia in the floor of the middle fossa by closing the defect with fascia lata. The swelling diminished in size after this operation but returned and, at a second operation by Mr. Bernard Harries, a further small hole was found and occluded with muscle. Following this the swelling disappeared.

These relatively small endaural hernias might, there- $\mathcal{O}$ fore, be associated with persistent purulent discharge $\mathrm{N}$ from the ear, cerebrospinal fluid otorrhoea, headache, and epilepsy - the latter in the absence of any previous history $\omega$ of abscess or generalized meningitis. A case in which a 
similar condition followed fracture of the skull base had been described by Andrew (1951). There might be no clinical evidence of intracranial infection or raised pressure in these patients. The dura usually formed a collar around the hernia and seldom covered the protrusion (Caboche, 1902). Repair should be undertaken through a craniotomy and, when the defect in the dura had been exposed by division of the surrounding adhesions, the area should be widely covered with fascia on the intradural surface.

(He expressed his indebtedness to Messrs. Julian Taylor and Bernard Harries for permission to report Cases 3 and 4. Case 3 was also under the care of Mr. C. S. Hallpike.)

\section{REFERENCES}

Andrew, W. F. (1951). Ann. Otol. (St. Louis), 60, 622. Caboche, H. (1902). Ann. Mal. Oreil. Larynx, 28, 278.

\section{Post-herpetic Neuralgia}

W. NOORDENBos (Amsterdam) reported the results of some investigations into this condition. The detailed results and conclusions are to be published at a later date.

\section{Ventriculo-jugular Anastomosis in the Treatment of Infantile Hydrocephalus}

T. B. Sikkens (Groningen) gave a preliminary report on a technique for making a shunt with a pump system between the lateral ventricle and superior vena cava in the treatment of hydrocephalus in infants. He noted that existing methods of treatment, such as shunts to the pleura or peritoneum, were unsatisfactory; removal of the choroid plexus and Torkildsen drainage operations often fell short of expectations and arachno-ureterostomy not only demanded the sacrifice of a kidney but also resulted in a continuous loss of electrolytes and cerebrospinal fluid. Direct drainage of cerebrospinal fluid into the venous system seemed to be the more physiological system to use.

Previous attempts at such a shunt had failed owing to back-flow of blood into the ventricular system and venous thrombosis. More recently, however, the construction of a shunt with a valve mechanism had been suggested and Nulsen and Spitz (1951) had reported a case treated by this method. Although pressure in the superior vena cava was nearly always lower than that in the lateral ventricle, nevertheless, some mechanism of preventing back-flow was desirable.

His apparatus consisted of a No. 6 rubber catheter which was inserted into the lateral ventricle via a parietooccipital burr hole. This was led down under the scalp and joined a repercussion valve. This consisted of a plexiglass tube, $1.5 \times 0.5 \mathrm{~cm}$., in which a small chamber was hollowed out and contained a stainless steel pellet of $1.5 \mathrm{~mm}$. diameter. Movement of the pellet would close the tube at the ventricular end thus preventing reflux of blood, but allowed passage of cerebrospinal fluid at the other end. There remained the risk that if the flow of cerebrospinal fluid was very slow or stopped, blood might diffuse up into the valve and block the tube.
This was overcome by adding a rubber pumping valve distal to the repercussion valve. It was arranged that the rubber pump lay over the mastoid process. This pump was then connected to an animal-tested polyethylene tube which was passed into the internal jugular vein which was then ligated. As this ligation stopped flow in the vein it was desirable that the tip of the tube should reach as far as the innominate vein or the superior vena cava. The polyethylene tube could then be kept patent by pumping cerebrospinal fluid through it. Pumping was started soon after the operation and carried out several times a day. The child's parents were instructed in the mechanism and, after leaving hospital, they carried out the pumping procedure daily.

So far six cases had been treated by this method. In two the results had been very satisfactory with a followup period of 10 months. In the other cases, the results had not been good. After studying the causes of the bad results he had decided to replace the polyethylene tube by a silicone-coated ivory tube. This would not only reduce the possibility of clot forming in and around the tube end but would also allow the position of the tube to be checked radiologically.

\section{REFERENCE}

Nulsen, F. E., and Spitz, E. B. (1951). Surgical Forum, 399. W. B Saunders, Philadelphia.

\section{Choroid Plexus Papilloma and its Relation to Hydrocephalus}

BERnARD Fairburn (Romford) presented a cáse of choroid plexus papilloma and discussed the relation of such tumours to the development of hydrocephalus. He noted that the association of hydrocephalus with papillomata of the choroid plexus had long been recognized. In two-thirds of the cases the tumour was in the third or fourth ventricle and obstruction was presumably the mechanism whereby the hydrocephalus was produced. In the remaining third, the tumour was in one of the lateral ventricles. It had often been suggested that oversecretion of cerebrospinal fluid was responsible in such cases for the ventricular dilatation but there was little objective evidence that this was, in fact, the operative factor.

Case Report.-A 15-month-old girl was treated for communicating hydrocephalus. A benign choroid plexus papilloma was found in the right lateral ventricle at necropsy and no other lesion was present which could have explained the hydrocephalus. The child presented with an acute rise of intracranial pressure. Posterior fossa exploration, carried out in the erroneous belief that there was a cerebellar tumour, gave very transient relief. Within two weeks the intracranial pressure had risen again and was not controlled by lumbar punctures and aspirations of the posterior fossa decompression. (These latter aspirations were followed by profuse leakage of cerebrospinal fluid along the needle-track for several hours.) Finally, continuous ventricular drainage was established through a catheter in the right frontal horn. During the three days that the catheter was in situ the volume of cerebrospinal fluid secreted was $500 \mathrm{ml}$., $400 \mathrm{ml}$., and $960 \mathrm{ml}$. respectively. The child showed clinical signs of dehydration during this period. 
He concluded that the findings in this case appeared to establish the fact that papillomata of the choroid plexus did secrete very large quantities of cerebrospinal fluid and that this was the mechanism of associated hydrocephalus.

\section{The Active and Progressive Nature of Certain Intracranial Herniations}

J. M. SMall (Birmingham) discussed the progressive nature of certain intracranial herniations. He noted that for centuries there had been interest in herniations of all kinds and their complications. Since the middle of the last century the knowledge that pupillary dilatation was associated with intracranial compression had been understood, but not until 1920 was the anatomy related to pupillary dilatation described. The passive nature of these intracranial herniations had been too easily accepted and their progression after release of compression ill understood.

Comparison was made between unilateral temporal compression with progressive coning and bilateral pupillary dilatation with mid-brain signs from vertical and polar pressure, showing this to be the difference between uncal herniation and descent of the mid-brain through the tentorial hiatus.

The progression of the tentorial cone syndrome with a low intracranial pressure following treatment of the initial compression was described and the value of tentorial division in such cases outlined.

Cases of impacted herniation beneath the falx were also described with expression of the need for division of the falx to free the persistent herniation.

Finally, the strangulation of tonsillar herniations was described in anomalies of the foramen magnum area with secondary vascular and compression changes in the stem.

\section{A Case of Meningioma in the Left Lateral and Third Ventricles}

M. WeERSMa (Rotterdam) discussed intraventricular meningiomas and presented the details of a personal case. He noted that such meningiomas were unusual in their lack of dural attachment and their incidence was low, Cushing and Eisenhardt (1938) reporting only 19 cases, Esbach (1943) 53 cases, all of the lateral ventricle, and Huang and Araki (1954) five cases.

Case Report.-Mrs. L.v. G., aged 54, came of a family with psychotic and psychopathic tendencies. The patient's mother had committed suicide. In July, 1957, she had many complaints which included fatigue, forgetfulness, and vague pains everywhere in the body. After an occasional attack of vomiting she complained of dizziness and she fell down while walking. She was admitted to the Department on September 17.

Examination showed bilateral papilloedema of two diopters, there was no impairment of visual acuity but a right homonymous hemianopia. Anisocoria was noted but pupil reactions and external ocular movements were normal. There was no nystagmus. A slight paresis of the right face, arm, and leg was noted and also some impairment of sensory perception on the right. All reflexes were present, there was no ataxia or Rombergism.
There was moderate arterial hypertension of $210 / 100 \mathrm{~mm}$. \ Hg.

Radiographs showed a calcified mass, about the size 8 of a hen's egg, in the central area of the head, mostly to the left of the midline. Ventriculography showed dilatation of both lateral ventricles and deformity of the left ventricle. Angiography showed slight displacement of the anterior cerebral artery to the right and widening $\stackrel{\rho}{S}$ of the loop of the pericallosal artery with compression
of the carotid siphon.

Operation was carried out on September 26, an approach being made to the tumour through the posterior $\overrightarrow{\bar{B}}$ parietal region. (It was noted that approach through the temporal region or by occipital lobectomy could also be used in these cases.) A highly calcified tumour $\left(80 \mathrm{~g}\right.$.) $\frac{}{\bar{O}}$ was removed, the major portion being in the lateral $\bar{c}$ ventricle but some portion of it lying in the third ventricle. $\widehat{\nabla}$ Histological examination confirmed the tumour as being $\varnothing$ a psammomatous arachnoidal meningioma.

After the operation there was marked oedema and bulging of the flap and a continuous fever but this. subsided after three weeks. Immediately there was a $\overrightarrow{\vec{H}}$ right hemiplegia and astereognosis, aphasia of the $\vec{\omega}$ amnesticreceptive type, alexia, and acalculia. There was $\stackrel{S}{?}$ loss of ability to recognize words and letters and to $\overline{3}$ discriminate between the right and left sides of the body $\overline{0}$ as well as external objects. The hemianopia was unaltered. is By persistent training there was steady improvement. $\vec{c}$ The patient could now walk without a stick and function $\omega$ in the right hand was also improved. The aphasia had $\mathrm{N}$ also recovered to some degree, though alexia was st present. Her mental state was not satisfactory, the being depressive and hysterical manifestations, part of which might be ascribed to her previous psychopathio personality. (It was noted with reference to the investiga tions of Goldstein (1943) that some reserve should be exercised in applying the term hysteria to patients wi⿺辶卬 parietal lesions.)

Ventriculograms and arteriograms were now near normal and the E.E.G. showed rhythmic 7 to $8 \mathrm{c}$./sec. $\infty$ activity and delta activity in the left parieto-temporal region.

\section{REFERENCES}

Cushing, H., and Eisenhardt, L. (1938). The Meningiomas. Charles C. Thomas, Springfield, Illinois.

Esbach, H. (1943). Allg. Path. path. Anat., 36, 185.

Goldstein, K. (1943). J. nerv. ment. Dis., 98, 148.

Huang, Y. S., and Araki, C. (1954). J. Neurosurg., $11,337$.

Pre-operative Dyshydration in Patients with Spaceoccupying Intracranial Processes

H. VERBIEST (Utrecht) gave a preliminary report on some investigations into fluid and electrolyte changes in patients with intracranial space-occupying lesions. $\mathrm{He}$ ? noted that in the past few years there had been a growing $\frac{5}{3}$ interest in the significance of disturbance of body fluids in neurosurgical patients. Most reports, however, had $ᄋ$ been concerned with changes in fluid pattern following trauma or surgery. The present report was concerned $ᄋ$ with pre-operative fluid disturbances in chronically ill patients suffering from intracranial space-occupying $\frac{D}{0}$ lesions. The investigation covered the period 1955-57 inclusive and comprised 254 patients. Patients with $\tilde{\sigma}$ unknown pathology or in whom investigations were incomplete had been excluded.

In this series pre-operative dyshydration occurred only $\mathrm{CN}^{\mathrm{W}}$ in lesions involving the cerebral hemispheres, cerebellum, O 
and fourth ventricle and tumours growing within the pons; there was one exception. In patients with lesions of the cerebellum and fourth ventricle there was a higher incidence of dyshydration in the lower age groups. In lesions of the cerebral hemispheres, $88.5 \%$ of 61 dyshydrated patients were older than 45 whereas $59 \%$ of 99 normally hydrated patients were younger than 45 .

There was no obvious relation between frequency of pre-operative dyshydration and the type of lesion so far as the posterior fossa was concerned. In lesions involving the cerebral hemispheres, the incidence was very high in intracerebral haematomas and glioblastoma multiforme, namely, $75 \%$ and $61 \%$ of cases respectively. The frequency of dyshydration was $50 \%$ in subdural haematomas, subdural empyemas, and cerebral abscesses. It varied between $30 \%$ and $40 \%$ in astrocytomas and metastases and was lowest $(12 \%)$ in meningiomas.

Frequent vomiting was the main cause of dyshydration in processes involving the cerebellum and fourth ventricle. In processes involving the cerebral hemispheres several factors were operative. These were, in order of frequency, mental symptoms which occurred in 60 of 61 patients and might have been responsible for insufficient spontaneous drinking or absence of the urge to drink; disturbances in drinking of administered water occurred in 42 or 61 cases and frequent vomiting occurred in 21 cases.

In frontal and temporal lobe processes the frequency of dyshydration was $52 \%$ and $49 \%$ and in parietal and occipital lesions $22 \%$ and $25 \%$ respectively.

The main patterns of fluid changes were: hyperosmolarity, hyperosmolarity and haemoconcentration, and haemoconcentration without hyperosmolarity. Azotaemia was present in $25 \%$ of dyshydrated patients with lesions in the posterior fossa and $79 \%$ of those with lesions in the cerebral hemispheres. In the whole series of 82 dyshydrated patients, acid-base equilibrium was disturbed in 42 cases, 33 presenting acidosis and nine alkalosis of metabolic origin. In the presence of dehydration the renal compensation for these disturbances is impaired.

In most cases of posterior fossa tumour, the fluid balance could be restored pre-operatively. Surgery of supratentorial lesions in dyshydrated patients had a very high mortality of $63 \%$ compared with a mortality of $21 \%$ in those admitted with a normal fluid balance and $17 \%$ in those who had their fluid disturbances corrected before operation. Sudden death during fluid replacement therapy occurred in two patients.

(This paper will be published in full in Archivum Europeum Neurochirurgiae.)

\section{Pathological Elements Appearing in the Urine after Cerebral Lesions}

R. C. KRUYT (Utrecht) presented a report on urine examinations in 462 patients who had had a neurosurgical operation or a head injury. In 48 of these abnormal constituents were found, including red cells, casts, and protein. Red cells appeared up to 100 per field and protein up to $20 \mathrm{~g}$./litre. These abnormalities appeared on the second or third day and persisted for five to 10 days. During this period the urea clearance was diminished.
In addition to these abnormalities 20 patients (50\%) had glycosuria, $29(60 \%)$ had a severe uraemia, and $33(70 \%)$ showed abnormalities in salt metabolism, either a salt retention with diminished excretion in the urine or an inability to retain salt, a salt-wasting syndrome. Pre-operatively none of the patients had abnormal elements in the urine. Of the 48 patients, 40 had a supratentorial lesion and of these $29(70 \%)$ had a lesion of the posterior orbital gyri. The remaining eight patients had a posterior fossa or high cervical lesion.

He noted that it was known that stimulation of the splanchnic and renal nerves produced a decrease of kidney volume with anuria and after stimulation the urine contained red cells and protein. Trueta had described a change in blood flow through the kidney after such stimulation in which the cortex became bloodless and the juxtamedullary region overfilled with blood (the Trueta mechanism or Oxford shunt). It was also known that puncture of the floor of the fourth ventricle produced polyuria, glycosuria, and increased salt excretion. Stimulation of the anterior hypothalamus had produced a decrease in urine flow which was abolished by denervation of the kidney. Microscopical examination showed tubular necrosis in these cases. Stimulation of the frontal lobes, especially of the posterior orbital gyri or area 13 (Brodmann), produced the Trueta effect in the kidney and at microscopic examination there was a lower nephron nephrosis. A lower nephron nephrosis had been found in two patients dying after electro-shock treatment and examination of the urine in a number of patients so treated had shown the presence of red cells, casts, and protein. After ventriculography, and especially after lumbar encephalography, protein and red cells had been found in the urine and there was a decreased excretion of urea and salt.

In the series reported none of the patients was in a state of shock of any duration. In five patients treated with A.C.T.H. the already existing urinary changes did not become either better or worse so that stress was probably not a deciding factor.

The high proportion of lesions in the posterior orbital gyri in this group suggested the existence of a specific influence of this region of the brain on blood flow in the kidney. That the stimulus was probably conveyed via the brain-stem and spinal cord was suggested by the urine changes in patients with posterior fossa or high cervical lesions.

\section{Compression of the Spinal Cord in Kyphosis and Kyphoscoliosis}

R. Malmros (Aarhus) presented a series of cases with kyphosis and kyphoscoliosis and spinal cord compression. In the Department of Neurosurgery in Aarhus, 18 patients had been operated on for this condition in the period 1943-58. Of these, 11 had a severe paraplegia, four a light or moderate paraplegia, and three bilateral spasticity and paralysis of one leg only. The patients could be divided into two groups according to age, a younger group in which the kyphosis was in most cases due to congenital malformations of the spine and an older group with mainly adolescent kyphosis (Scheuermann). In two cases the kyphosis was due to an old tuberculous 
spondylitis. Only the facts related to the younger group were presented.

He noted that compression of the spinal cord with paraplegia was rare in kyphoscoliosis, most authors having reported only single cases. McKenzie and Dewar (1949) collected 36 cases from the literature and added five of their own. However, in one of their cases compression was due to a fibrous band and syringomyelia was later demonstrated in another. More single cases had been reported since then.

In his series there were seven males and three females. The age distribution was: 8-9 years two cases, 13-15 years three cases, 17-18 years two cases, and 21-24 years three cases. The clinical picture was very uniform. The symptoms were slowly progressive, starting with a staggering gait due to increasing spasticity and ending in paraplegia. Diminution of sensibility over the trunk and legs occurred and was followed, usually later in the course, by sphincter disturbance in the form of precipitate micturition or, sometimes, urinary retention.

Paresis usually began in the feet and spread upwards. The course was one of slow progression so that within six to 12 months there might be complete motor and sensory loss but still relatively little sphincter disturbance.

Another characteristic feature of the disease was that there was no pain during its progression. There might be numbness and tingling in the legs but the root pain so often seen in patients with spinal tumour or spondylitis was absent.

Of the 10 patients, nine had severe paraplegia and were confined to bed. One had bilateral spasticity and paraplegia of the legs, moderate in one and slight in the other, but was able to walk. The duration of symptoms was two to four months in two cases, five to six months in three, and 12 to 18 months in five cases.

All patients were treated by surgical decompression. An extensive laminectomy was performed at the apex of the kyphosis. The dura was found to be displaced anteriorly in the spinal canal and in eight patients there was a space between dura and laminae, 12 to $20 \mathrm{~mm}$. wide, which was filled with fatty tissue. The dura was opened longitudinally and, as the cord prolapsed into the incision, transverse cuts were made and the edges sewn up.

Seven patients made a complete recovery (follow-up periods one to $13 \frac{1}{2}$ years). In three cases, the results were poor. One boy of 17 did not improve and died two years later. One boy of eight improved at first but symptoms recurred five years later. Re-operation six years after the original operation revealed extensive scar formation and adhesions in the spinal canal at the apex of the kyphosis. He was still living but with a severe paraplegia. The third case, a boy of nine years of age, had improved only slowly over a period of two years.

He concluded that cord compression was due to pressure exerted by the dura. This was bent over the kyphosis and behaved like an inelastic tube. The posterior dura approached the anterior dura and the cord was squeezed between.

\section{REFERENCE}

McKenzie, K. G., and Dewar, F. P. (1949). J. Bone Jt Surg., 31-B, 162.
Radiological Aspects of Functional Listhesis of the Spine

P. Hanraets (Wassenaar) discussed the radiological appearances of a functional form of spondylolisthesis. He mentioned the occurrence of apparent retroposition of a vertebra due to congenital antero-posterior shortening of the body and concave posterior margin to the sacrum but did not discuss these in detail. In a group of patients true spondylolisthesis with lysis of the arch occurred in $4 \%$, pseudo spondylolisthesis with no lysis of the arch but disc narrowing in $6 \%$, and functional listhesis in $7 \%$. In this latter group certain mechanisms came into operation which disorganized the function of the lumbar spine so radically that listhesis might ensue, irrespective of whether the intervening disc had degenerated or not. The cause of listhesis appeared to be disease at levels other than at which displacement occurred. Indeed, the very existence of listhesis might not be suspected unless spinal function was examined radiologically.

This condition occurred when the spine was inadequate functionally with the result that the lumbar region was strained and the discs and ligaments put under such strain that, in order to provide the required curvature, vertebral bodies began to slip in relation to each other. One in every four of such minor displacements was multiple.

In this group of functional listhesis the causativ $\circlearrowright \omega$ factors found were: the latter months of pregnancy a pendulous abdomen; chronic postural anomalies else $\stackrel{\rho}{\rho}$ 을 where in the spine such as thoracic kyphoscoliosis; क wide angle between lumbar spine and sacrum; any chroniç back strain. Displacements in this group were morê prone to occur in the presence of asthenia, a previous extensive laminectomy and dystrophia musculorum progressiva.

The extent of displacement might be only a few milli? metres and not apparent for some time yet produce symptoms due to the effect of opposing forces. This was the probable mechanism of low back pain in the overstrained weak back. The beneficial effect of rest was due to the opportunity given to muscles to recuperate and so prevent the functional listhesis from coming into operation.

A second mechanism producing functional listhesis was any local area of fixation in the lumbar spine. Local limitation of movement had to be compensated for at other levels by vertebral displacement and this usually took place one segment above the fixed area. Where possible the displacement took place in an area where the strain was greatest, i.e., one segment above, and at a place where the spine was most mobile. The discs were normal in the affected area and not primarily degenerative. This mechanism had been seen in association with the following conditions: Trauma at thoraco-lumbar level; disc herniation at high lumbar level; congenital synostosis of two lumbar vertebrae with long sacrum and transitional vertebrae; after disc operations and fusion operations and after infection; and in functional synostosis with disc herniation associated with muscle spasm and obliteration of the normal lumbar lordosis.

Low back pain in this second group was brought about primarily by the effect of opposing forces though root symptoms might also occur at a later date. 
Every such displacement limited space relatively though it need not necessarily cause symptoms or signs. Their occurrence depended both on the degree of displacement and on the existing space within the canal. The coincidence of a functional listhesis with wide but oblate shape of the spinal canal in the lumbo-sacral region was mentioned. Such a shaped canal was a manifestation of a constitutional type and not identical with the wide canal seen in the thoracic and high lumbar regions (Elsberg, Dyke, and Brewer, 1934; Jefferson, 1955). The combination with spina bifida might be pertinent though a predisposition to relative stenosis had not been described (Walker, 1944). It might be inferred that the vertebral column with wide flat canal is predisposed to functional listhesis and where the two exist together the stenosis might be such as to effect root compression.

\section{REFERENCES}

Elsberg, C. A., Dyke, C. G., and Brewer, E. D. (1934). Bull. neurol. Inst., N.Y., 3, 395 .

Jefferson, A. (1955). J. Neurol. Neurosurg. Psychiat., 18, 305.

Walker, A. E. (1944). Amer. J. Roentgenol., 52, 571.

Hyperthrophic Cervical Pachymeningitis in Three Sisters

M. P. A. M. DE GROOD (Tilburg) described the occurrence of cervical pachymeningitis in three sisters. They were the first, fourth, and eleventh children of a large family of 18. Symptoms and signs appeared in adult life and comprised a spastic paraparesis with claw hands. Sensory and sphincteric disturbances were absent in two of the cases. They all exhibited evidence of a dysraphic state with many congenital anomalies. These included dwarfism, short straight neck, broad face, high scapula, high palate, and kyphosis. Two sisters had a congenital systolic murmur in the heart and the third a mitral stenosis. Congenital narrowing of the cervical canal was excluded by radiography and myelography which showed, in all cases, a hold-up in the high cervical region. Operation was undertaken in each case and disclosed compression of the cervical cord by hypertrophic dura mater.

He noted that, although cases attributable to tuberculosis, syphilis, and trauma were recognized, idiopathic cases remained in which no cause could be found. Syringomyelia had sometimes been found in association. In the present group, as no other cause could be found, it was concluded that the hypertrophic pachymeningitis was a manifestation of the dysraphic state. 